\title{
CSE (Ceftriaxone+ Sulbactam+ Disodium Edta): A Possible Solution to the Global Antimicrobial Resistance Pandemic
}

\author{
Shalini Upadhyay* (D), Anita Chakravarti, Tanisha Bharara and Sushmita Yadav
}

Department of Microbiology, SGT University, Budhera, Gurugram - 122505, Haryana, India.

\begin{abstract}
The menace of multidrug resistance among bacterial infections is an issue of global public health. Treatment of these superbugs with first line antibiotics is associated with significant treatment failure leading to increased mortality and morbidity. Carbapenems and polymyxins are the saviour antibiotics in case of such infections. But the problem is compounded when these antimicrobials also fail. The addition of beta-lactamase inhibitor like sulbactam and disodium ethylenediaminetetraacetic acid broaden the in vitro antibacterial action of ceftriaxone. This novel combination has been found to be effective in most of the drug resistant bacterial strains.
\end{abstract}

Keywords: Antibiotic adjuvant, Antibiotic resistance breakers, Carbapenem resistance, Multidrug resistance

(C) The Author(s) 2020. Open Access. This article is distributed under the terms of the Creative Commons Attribution 4.0 International License which permits unrestricted use, sharing, distribution, and reproduction in any medium, provided you give appropriate credit to the original author(s) and the source, provide a link to the Creative Commons license, and indicate if changes were made. 


\section{INTRODUCTION}

A broad spectrum of infections ranging from pyaemia to bacteraemia are caused by gram-negative bacteria. Such infections are a substantial burden especially in critical care units $^{1}$. There has been a significant increase in multidrug resistant (MDR) bacterial strains all over the world, more so in South-East Asian countries, including India ${ }^{1,2}$. Carbapenems are structurally stable against common resistant mechanisms, so they are considered to have a frontline role in the treatment regime for MDR micro-organisms. However, with the recent upsurge of carbapenemresistant bacteria in the last few years, the efficacy of carbapenems in treating these drug resistant strains has been undermined ${ }^{3,4}$.

CSE, an antibiotic adjuvant entity (AAE) is combination of ceftriaxone, sulbactam and disodium ethylene-diamine-tetraacetic acid (EDTA). It displays a broad spectrum of antibiotic activity against gram negative infections. This has been proposed to be an alternative for management of gram negative MDR threat. The mechanism of action of CSE has been depicted in the Fig. $1^{5,6,7}$. Various clinical trials of CSE have showed it's efficacy towards many MDR micro-organisms as compared to beta lactambeta lactamase inhibitor combination and carbapenems. These studies have shown that it can act as a potential alternative treatment for reducing the burden on use of last resort antibiotics $^{8-11}$. After phase III clinical trials, this drug got approved by Indian FDA and thereafter has been marketed in India for the treatment of MDR infections. CSE has been approved by Drug Controller General of India and is being used since more than 5 years now ${ }^{1,5,8}$. Since the rural population is less explored with respect to antibiotic resistance, we aims to analyse the susceptibility of CSE versus cephalosporins and carbapenems among gram negative bacteria isolated from various microbiological specimens in a rural setup. This knowledge will help clinicians in deciding whether CSE can be used when the above antimicrobials for treatment of MDR pathogens are not susceptible and also to save the last resort antibiotics like carbapenems and polymyxins.

\section{MATERIAL AND METHODS Study design and study population}

This is an observational, cross-sectional study conducted at a multi-speciality hospital of North India catering to rural population. The samples received in Microbiology laboratory for culture \& antimicrobial susceptibility testing on clinical suspicion of infection were processed. One hundred twenty clinical isolates having growth of gram-negative bacteria from different clinical samples were collected from July 2019 to November 2019. All patient details were

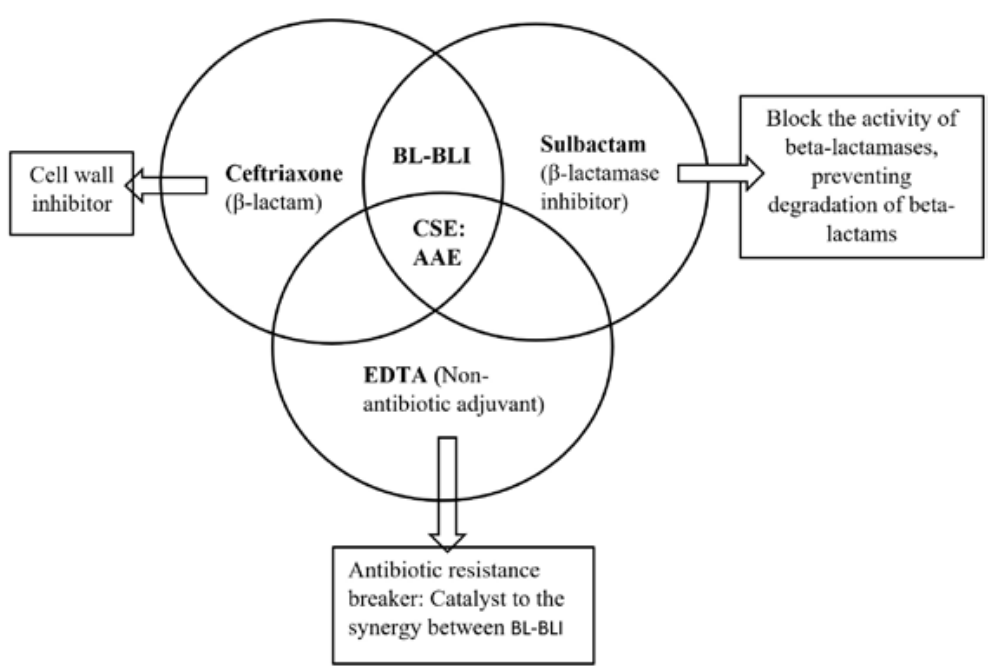

Fig. 1. The mechanism of action of CSE

Note: CSE: Ceftriaxone+Sulbactam+EDTA, BL-BLI: beta lactam-beta lactamase inhibitor, AAE: antibiotic adjuvant entity, EDTA: ethylene-diamine-tetraacetic acid. 
anonymized, coded by randomization and delinked from any identity of the patients.

\section{Sample processing}

On clinical suspicion of infection at any site, appropriate specimens were sent to the Microbiology laboratory. The blood specimens received were inoculated into blood culture bottles and aerobically incubated in the BacT/ALERT 3 D system (bioMerieux). When machine flashed positive for the bottle, Gram's stain was prepared. In case of urine specimen, it was inoculated on cysteine lactose electrolyte deficient (CLED) culture media for semiquantitative culture. A colony count of $10^{4}-10^{5}$ was considered significant for urine culture. For all other specimens, subculture was done on blood agar and MacConkey agar. Antimicrobial susceptibility test (AST)

AST was performed by Kirby-Bauer disc diffusion method on MHA (Mueller Hinton Agar). Inoculum with turbidity of the 0.5 McFarland standard from the test strain was inoculated with a sterile swab on MHA plates. Within 15 minutes, discs of ampicillin $(10 \mu \mathrm{g})$, ceftriaxone $(30 \mu \mathrm{g})$, amikacin $(30 \mu \mathrm{g})$, piperacillin-tazobactam $(100 / 10 \mu \mathrm{g})$, gentamicin $(10 \mu \mathrm{g})$, cotrimoxazole $(1.25 / 23.75 \mu \mathrm{g})$, ciprofloxacin $(5 \mu \mathrm{g})$, ertapenem $(10 \mu \mathrm{g})$ and CSE $(45 \mu \mathrm{g})$ were applied to the MHA plates and incubated. Zone of inhibition was measured in $\mathrm{mm}$ (millimetre) after incubation at 34- $36^{\circ} \mathrm{C}$ for $16-20$ hours.

\section{Quality control}

Escherichia coli (E. coli) ATCC 25922, Klebsiella pneumoniae (K. pneumoniae) ATCC 700603 and Pseudomonas aeruginosa ( $P$. aeruginosa) ATCC 27853 were the quality control strains.

Interpretation

The Clinical and Laboratory Standards Institute guidelines (CLSI) 2019 was used to characterize the isolates into susceptible, intermediate and resistant ${ }^{12}$. MDR microorganisms were those which showed resistance to three or more classes of antimicrobials.

\section{Statistical analysis}

Data was analysed using SPSS software Version 20.0. A p-value $<0.05$ was considered statistically significant. Comparative statistical analysis was performed using chi-square test.

\section{RESULTS}

A total of 1483 specimens with suspicion of bacterial infection were received in the microbiology laboratory throughout the study period. The cultures came out to be positive in 437 samples, out of which 182 were Gram negative bacteria. One twenty of the 182 culture were

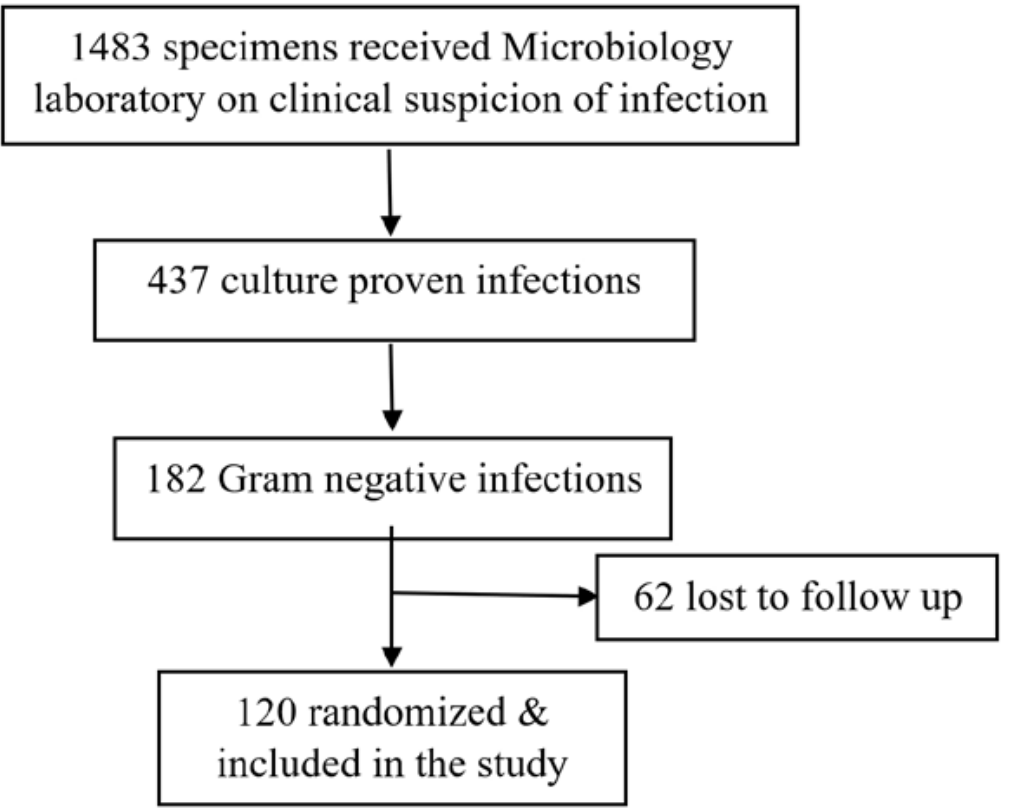

Fig. 2. The specimen inclusion flowchart 
included in the study as the details of the rest could not be traced (Fig. 2). Of the 120 samples included in the study, majority were urine samples, followed by pus, blood, and others which included body fluids and endotracheal secretion.

The most common age group was 2060 years and females (69/120) outnumbered males though the difference was not statistically significant ( $p$ value $<0.104$ ). Around $72 \%$ specimens were from outpatient department while $23 \%$ were inpatients ( $p$ value $<0.0001$ ). The most common specimen was urine $(51.7 \%)$, followed by pus (26.7\%) and blood (12.5\%). Enterobacteriaceae

Table 1. Characteristics of the study population

\begin{tabular}{lc}
\hline Characteristics & $\mathrm{n}(\%)(\mathrm{N}=120)$ \\
\hline Gender & $51(42.5)$ \\
Male & $69(57.5)$ \\
Female & \\
Age (years) & $32(26.7)$ \\
$0-20$ & $59(49)$ \\
$20-60$ & $29(24)$ \\
$>60$ & \\
Location & $86(71.7)$ \\
IPD (In-patient department) & $34(28.3)$ \\
OPD (Out-patient department) & \\
Diagnosis & $62(51.7)$ \\
Urinary tract infection & $32(26.7)$ \\
Pyaemia & $15(12.5)$ \\
Bacteraemia & $11(91.7)$ \\
Others & \\
Gram negative pathogens isolated & $59(49)$ \\
E. coli & $23(19)$ \\
K. pneumoniae & $16(13)$ \\
Other Enterobacteriaceae & $13(11)$ \\
Pseudomonas spp. & $9(7)$ \\
Acinetobacter spp. & \\
&
\end{tabular}

were the most common among the Gram-negative microorganisms ( $p$ value $<0.0001,95 \% \mathrm{Cl}=0.47-0.9$ ) with E. coli (49\%), K. pneumoniae (19\%), followed by Pseudomonas spp. (11\%) and Acinetobacter spp. (7\%) (Table 1).

The susceptibility of $E$. coli isolates was $85 \%$ to amikacin and $76 \%$ each to ertapenem and gentamicin. K. pneumoniae isolates were more resistant than other Enterobacteriaceae with $78 \%$ susceptibility to amikacin, $73 \%$ to ertapenem and $70 \%$ to gentamicin. The most resistant among all the Gram negative bacteria were Acinetobacter spp. with $78 \%$ to amikacin and $65 \%$ to ertapenem (Table 2). MDR strains were detected in $24 \%$ of $E$. coli isolates, $27 \% \mathrm{~K}$. pneumoniae, $29 \%$ Pseudomonas spp. and $35 \%$ of Acinetobacter spp. There is statistically significant difference between sensitivity of beta-lactam (ceftriaxone) $\&$ CSE ( $p$ value $<0.0001$ ) and also beta-lactam-beta lactamase inhibitor \& CSE ( $p$ value $<0.0005)$.

\section{DISCUSSION}

In the present study female preponderance can be explained by the fact that majority of the samples were from suspected UTI patients which is commoner in females similar to other Indian studies ${ }^{13,14}$. The middle age group of 20-60 years is most implicated in infections as they are the earning members of the family. This population is most exposed to the environmental risk factors and so vulnerable to minor infections. The older age group of more than 60 years if infected have severe complications that may lead to prolonged hospital stay and thus exposure to hospital flora leading to life threatening infections. The members of Enterobacteriaceae (especially $E$. coli) were the most common pathogens since UTI

Table 2. The antibiotic susceptibility profile of Gram negative bacteria

\begin{tabular}{lllllllll}
\hline \multicolumn{1}{c}{ GNB } & \multicolumn{7}{c}{ Antibiotic susceptibility (\%) } \\
\cline { 2 - 8 } & AMP & CTR & AK & PIT & GEN & COT & CIP & ETP \\
\hline E. coli & 15 & 32 & 85 & 71 & 76 & 46 & 49 & 76 \\
K. pneumoniae & 13 & 26 & 78 & 65 & 70 & 43 & 47 & 73 \\
Other Enterobacteriaceae & 19 & 35 & 87 & 81 & 81 & 44 & 50 & 88 \\
Pseudomonas spp. & 15 & 26 & 77 & 61 & 69 & 38 & 46 & 71 \\
Acinetobacter spp. & 11 & 22 & 78 & 56 & 56 & 33 & 44 & 65 \\
\hline
\end{tabular}

Note: GNB: Gram negative bacteria, AMP-Ampicillin, CTR-Ceftriaxone, AK-Amikacin, PIT-Piperacillin-tazobactam, GEN-Gentamicin, COT-Cotrimoxazole, CIP-Ciprofloxacin, ETP-Ertapenem. All the percentages have been rounded off to whole number.

Journal of Pure and Applied Microbiology 


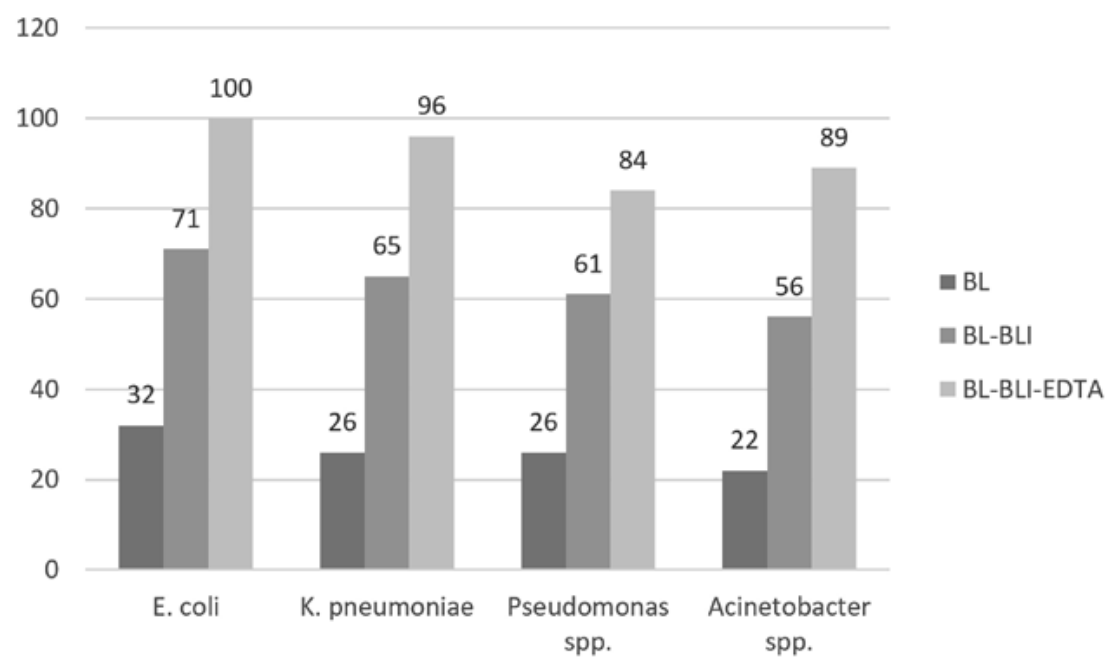

Fig. 3. Comparative susceptibility of CSE with beta lactam and beta lactam-beta lactamase inhibitor Note: BL (beta lactam, Ceftriaxone), BL-BLI (beta lactam-beta lactamase inhibitor, Piperacillin-tazobactam), CSE: Ceftriaxone+Sulbactam+EDTA (BL-BLI-EDTA combination), EDTA: ethylene-diamine-tetraacetic acid.

was the commonest infection similar to isolation rates in other studies. The resistant strains were mostly isolated from the in-patients admitted in wards and intensive care units ${ }^{14-17}$.

There has been sharp rise in MDR bacterial infections, especially against common antimicrobials such beta-lactams which are the first line of treatment. Global trends of resistance as assessed by WHO show $16-68 \%$ third-generation cephalosporin resistance among $E$. coli while 34$81 \%$ among $K$. pneumoniae in South-East Asian Region $^{18}$. In our study, Gram-negative bacteria showed $65-78 \%$ resistance to cephalosporins and $12-35 \%$ resistance for carbapenem while there was good sensitivity to CSE. The most resistant pathogen was Acinetobacter spp. while it was K. pneumoniae among the members of Enterobacteriaceae that showed high resistance. Indian studies on gram negative bacterial infections have reported high rates of resistance for $\beta$-lactams, $\beta$-lactams$\beta$-lactamase inhibitors and rapidly rising trend for carbapenems $s^{19,20}$. Irrational and overuse of the reserve antibiotics like carbapenems and colistin which are recommended only in MDR cases ${ }^{21}$ has led to their increased resistance. This pattern is a serious concern and is now very evident in Indian scenario ${ }^{22}$. Considering the above situation newer strategies are needed to treat MDR infections while sparing the last resort antibiotics as development of newer drugs is time consuming and exhaustive. To tackle the rising menace of MDR, the collective aim should be on narrowing down the number of antibiotics use to essential only and de-escalating the broad spectrum antibiotics as per the susceptibility report. The currently effective high end antibiotics should be preserved for clinical use in critical cases.

ARBs (antibiotic resistance breakers) or antibiotic adjuvants are non-antibiotic moieties without any direct antimicrobial activity but in combination with currently failing antibiotic agents, they can overcome various resistance barriers ${ }^{23}$. EDTA augments the penetration of drug by enhancing porosity and chelates divalent ions in extracellular polysaccharides \& bacterial cells thus damaging the microbial biofilm, resulting in reduction of MIC. Further, CSE modulates the overexpression of efflux pumps and curtails the transfer of resistant plasmids, thus preventing the spread of resistance. ${ }^{24-26}$. Results of our study show good in vitro sensitivity of CSE for most of the Gram negative bacteria commonly causing MDR infections $^{27,28}$. The safety profile of CSE has been found to be similar to that of ceftriaxone alone ${ }^{29}$.

There are a few limitations of our study. First, it was a single centre study. Second, clinical details of patients could not be included in the study as that would require ethical clearance. The 
strength of this study lies in the fact that it studies rural population of a developing country which is less explored. Also, strict, protocol-driven practices for sample collection were followed, reducing any chance of contamination.

Repurposing drugs using ARBs not only overcomes many of the barriers to developing new antibiotics but it could also salvage many of the existing antibiotics for the future generations.

\section{ACKNOWLEDGMENTS}

None.

\section{CONFLICT OF INTEREST}

The authors declare that there is no conflict of interest.

\section{FUNDING}

None.

\section{AUTHORS' CONTRIBUTION}

All authors listed have made a substantial, direct and intellectual contribution to the work, and approved it for publication.

\section{ETHICS STATEMENT}

Not applicable.

\section{DATA AVAILABILITY}

All datasets generated or analysed during this study are included in the manuscript.

\section{REFERENCES}

1. Chaudhary M, Mir MA, Ayub SG, Protocol 06 Group1. Safety and efficacy of a novel drug elores (ceftriaxone + sulbactam + disodium edetate) in the management of multi-drug resistant bacterial infections in tertiary care centers: a post-marketing surveillance study. Braz J Infect Dis. 2017;21(4):408-417. doi: 10.1016/j. bjid.2017.02.007

2. WHO. Antimicrobial resistance: global report on surveillance2014. WHO; 2016. Available from: http://www.who.int/drugresistance/documents/ surveillancereport/en/ [accessed March 17, 2020].

3. Gupta E, Mohanty S, Sood S, Dhawan B, Das BK, Kapil A. Emerging resistance to carbapenems in a tertiary care hospital in north India. Indian J Med Res. 2006;124(1):95-98.

4. Kanj SS, Kanafani ZA. Current concepts in antimicrobia therapy against resistant Gram-negative organisms: extended-spectrum beta-lactamase-producing Enterobacteriaceae, carbapenem-resistant Enterobacteriaceae, and multidrug-resistant Pseudomonas aeruginosa. Mayo Clin Proc.
2011;86(3):250-259. doi: 10.4065/mcp.2010.0674

5. Fatima N, Shameem M, Khan HM, Shukla I. Determination of susceptibility breakpoints of a novel Antibiotic Adjuvant Entity CSE1034 in extended spectrum beta lactamases and metallo betalactamases producing resistant Enterobacteriaceae. $J$ Microbiol Antimicrob. 2017;3(1):25-33.

6. Chaudhary M, Payasi A. A randomized, open-label, prospective, multicenter phase-III clinical trial of Elores in lower respiratory tract and urinary tract infections. J Pharm Res. 2013;6(4):409-414. doi: 10.1016/j. jopr.2013.04.011

7. Gupta R. Antibiotic adjuvant therapy for multi-drug resistant carbapenemases producing Klebsiella pneumoniae associated sepsis: a case study. J Clin Diagn Res. 2016;10(4):DD08-DD09. doi: 10.7860/ JCDR/2016/17544.7697

8. Bhatia P. Alternative empiric therapy to carbapenems in management of drug resistant Gram negative pathogens: a new way to spare carbapenems. Res $J$ Infect Dis. 2015;3:2.10. doi: 10.7243/2052-5958-3-2

9. Chaudhary M, Payasi A. Clinical, microbial efficacy and tolerability of Elores, a novel antibiotic adjuvant entity in ESBL producing pathogens: Prospective randomized controlled clinical trial. J Pharmacy Res. 2013;7(4):275280. doi: 10.1016/j.jopr.2013.04.017

10. Rajpurohit H, Kumar VBM, Sharadamma KC, Radhakrishna PM. Comparative study of antimicrobial activity of ceftriaxone in combination with sulbactam and tazobactam using disc diffusion method. Int Res J Pharmacy. 2012;3:331-334.

11. Mir MA, Chaudhary S, Payasi A, Sood R, Mavuduru RS, Shameem M. Ceftriaxone+ Sulbactam+ Disodium EDTA Versus Meropenem for the Treatment of Complicated Urinary Tract Infections, Including Acute Pyelonephritis: PLEA, a Double-Blind, Randomized Noninferiority Trial. Open Forum Infect Dis. 2019;6(10):ofz373. doi: 10.1093/ofid/ofz373

12. Clinical and Laboratory Standards Institute. Performance standards for antimicrobial susceptibility testing; twenty-ninth informational supplement. CLSI document M100-S29. Wayne, PA 19087 USA, 2019.

13. George CE, Norman G, Ramana GV, Mukherjee D, Rao T. Treatment of uncomplicated symptomatic urinary tract infections: Resistance patterns and misuse of antibiotics. J Family Med Prim Care. 2015;4(3):416421.doi: 10.4103/2249-4863.161342

14. Prakash D, Saxena RS. Distribution and antimicrobial susceptibility pattern of bacterial pathogens causing urinary tract infection in urban community of Meerut city, India. ISRN Microbiol. 2013:1-13 doi: 10.1155/2013/749629

15. Somashekara SC, Deepalaxmi S, Jagannath N, et al. Retrospective analysis of antibiotic resistance pattern to urinary pathogens in a tertiary care hospital in South India. J Basic Clin Pharm. 2014;5(4):105-108. doi: 10.4103/0976-0105.141948

16. Singhal A, Sharma R, Jain M, Vyas L. Hospital and community isolates of uropathogens and their antibiotic sensitivity pattern from a tertiary care hospital in North West India. Ann Med Health Sci Res. 2014;4(1):51-56. doi: 10.4103/2141-9248.126611 
17. Kant S, Lohiya A, Kapil A, Gupta SK. Urinary tract infection among pregnant women at a secondary level hospital in Northern India. Indian J Public Health. 2017;61:118-123.

18. WHO. Prioritization of pathogens to guide discovery, research and development of new antibiotics for drugresistant bacterial infections, including tuberculosis. Geneva: World Health Organization; 2017(WHO/EMP/ IAU/2017.12). (Licence: CC BY-NC-SA 3.0 IGO.)

19. Niranjan V, Malini A. Antimicrobial resistance pattern in Escherichia coli causing urinary tract infection among inpatients. Indian J Med Res. 2014;139:945-48.

20. Satras KB. Prevalence and Susceptibility Analysis of Gram Negative Pathogens in Super Specialty Tertiary Care Centers, Pune in India from January 2018- January 2019. Microbiology Research Journal International. 2019;28(6):1-9. doi: 10.9734/mrji/2019/v28i630150

21. Deshpande P, Rodrigues C, Shetty A, Kapadia F, Hedge A, Soman R. New Delhi Metallo-beta lactamase (NDM1) in Enterobacteriaceae: treatment options with carbapenems compromised. J Assoc Physician India. 2010;58:147-149.

22. Ghafur AK. Can India be the Wing Commander in the Global fight against Antimicrobial Resistance? J Assoc Physicians India. 2012;60:42-43.

23. Laws M, Shaaban A, Rahman KM. Antibiotic resistance breakers: current approaches and future directions.
FEMS Microbiology Reviews. 2019;43(5):490-516. doi: 10.1093/femsre/fuz014

24. Chaudhary M, Payasi A. Role of EDTA and CSE1034 in curli formation and biofilm eradication of Klebsiella pneumoniae: a comparison with other drugs. J Antibiot. 2012;65:631-633. doi: 10.1038/ja.2012.82

25. Chaudhury M, Kumar S, Payasi A. Role of CSE1034 in E. coli biofilm destruction. J Microb Biochem Technol. 2013a;5:54-8.

26. Chaudhury M, Payasi A. Inhibition of metalo beta lactamases by Elores. J Antimicrob. 2013B;128:177-82.

27. Chaudhary M, Kumar S, Payasi A. In vitro susceptibilities of clinical isolates of Escherichia coli and Klebsiella species to CSE1034 and other beta-lactams. The J Antibio. 2013;66:495-497. doi: 10.1038/ja.2013.29

28. Kumar M, Chaudhary S, Makkar DK, Garg N, Chugh S. Comparative antimicrobial efficacy evaluation of a new product Elores against meropenem on gram-negative isolates. Asian J Pharmaceut Clin Res. 2015;8:1-4.

29. Chaudhary M, Ayub SG, Mir MA, Members of Study Group. CSE-1034 versus Ceftriaxone: Efficacy and Safety Analysis from a Randomized, Openlabeled Phase III Study in Complicated Urinary Tract Infections. J Glob Infect Dis. 2018;10(4):188-195. doi: 10.4103/jgid.jgid_98_17 